\title{
Intervenção educativa sobre diabetes mellitus em pacientes atendidos em Nova Iguaçu-RJ
}

\author{
Educational intervention on diabetes mellitus in patients \\ attended in Nova Iguaçu-RJ
}

\section{Intervención educativa sobre diabetes mellitus en pacientes atendidos en Nova Iguaçu-RJ}

\author{
Emanuel Inocêncio Ribeiro da Silva ${ }^{1}$, Hellen de Souza Neves Martins ${ }^{1}$, \\ Renata Rodrigues Teixeira de Castro ${ }^{2}$, Adalgiza Mafra Moreno ${ }^{3}$, Paula \\ Guidone Pereira Sobreira ${ }^{4}$, Rúbia Walquíria de Carvalho ${ }^{5}$, Emílio \\ Mistsunao Takana ${ }^{6}$, Raphael Coelho de Almeida Lima ${ }^{1}$, \\ Roberta Rodrigues Teixeira de Castro ${ }^{7}$
}

\author{
1.Médico. Universidade Iguaçu - UNIG, Nova Iguaçu-RJ, Brasil. \\ 2.Médica, Doutora. Marcílio Dias. Universidade Iguaçu - UNIG, Nova Iguaçu-RJ, Brasil. \\ 3.Fisioterapeuta. Doutora. Universidade Iguaçu - UNIG, Nova Iguaçu-RJ, Brasil. \\ 4.Psicóloga. Mestre. Universidade Iguaçu - UNIG, Nova Iguaçu-RJ, Brasil. \\ 5.Administradora. Mestre. Instituto Nacional do Câncer - Inca. Rio de Janeiro-RJ, Brasil. \\ 6. Estudante de medicina. Universidade Iguaçu - UNIG, Nova Iguaçu-RJ, Brasil. \\ 7.Médica Veterinária. Doutora. Instituto Nacional do Câncer - Inca. Universidade Iguaçu - UNIG, Nova \\ Iguaçu-RJ, Brasil.
}

\section{Resumo}

Objetivos. Analisar os efeitos da educação em saúde em pacientes com Diabetes Mellitus atendidos no Hospital Geral de Nova Iguaçu-RJ, visando ampliar o conhecimento sobre o cuidado integral e interdisciplinar a respeito da doença. Método. Foi elaborado questionário com perguntas contemplando conhecimento sobre a doença, fatores de risco, formas de controle e tratamento e percepção de risco. Sua aplicação foi realizada em 77 pacientes, em três momentos distinto, pré e pós a realização das palestras e via contato telefônico três meses após a realização das mesmas. Foi utilizado o Teste de McNemar para identificar a retenção do conhecimento pelos pacientes à curto e médico prazo. Resultados. Houve mudança significante em $100 \%$ das respostas dos participantes, imediatamente após as oficinas educativas, demonstrando ampliação no conhecimento sobre a doença. Três meses após a intervenção educativa não houve mudança significativamente estatística, demonstrando que a retenção do aprendizado foi eficaz a ponto de perdurar nesse intervalo de tempo. Conclusões. Neste estudo, o intervalo de tempo entre a aplicação do questionário e a realização do recurso educacional não promoveu perda de aprendizado pelos participantes.

Unitermos. Diabetes Mellitus; Educação em Saúde; Autocuidado

\begin{abstract}
Objectives. To analyze the effects of health education on patients with Diabetes Mellitus treated at health centers in the municipality of Nova Iguaçu-RJ, aiming to expand knowledge about comprehensive and interdisciplinary care regarding the disease. Method. A questionnaire was prepared with questions that included knowledge about the disease, risk factors, forms of control and treatment and risk perception. Its application was carried out in 77 patients, in three different moments, before and after the lectures and via telephone contact three months after the lectures. The McNemar test was used to identify the retention of knowledge by patients in the short and medical term. Results. There was a significant change in $100 \%$ of the participants' responses, immediately after the educational workshops,
\end{abstract}


demonstrating an increase in knowledge about the disease. Three months after the educational intervention, there was no statistically significant change, demonstrating that the retention of learning was effective to the point of lasting in that interval of time. Conclusions. In this study, the time interval between the application of the questionnaire and the realization of the educational resource did not promote loss of learning by the participants.

Keywords. Diabetes Mellitus; Health education; Self care

\section{Resumen}

Objetivos. Analizar los efectos de la educación para la salud en pacientes con Diabetes Mellitus atendidos en los centros de salud del municipio de Nova Iguaçu-RJ, con el objetivo de ampliar el conocimiento sobre la atención integral e interdisciplinaria de la enfermedad. Método. Se elaboró un cuestionario con preguntas que incluían conocimientos sobre la enfermedad, factores de riesgo, formas de control y tratamiento y percepción del riesgo. Su aplicación se llevó a cabo en 77 pacientes, en tres momentos diferentes, antes y después de las conferencias y vía contacto telefónico tres meses después de las conferencias. El Test de McNemar se utilizó para identificar la retención de conocimientos por parte de los pacientes a corto y plazo médico. Resultados. Hubo un cambio significativo en el $100 \%$ de las respuestas de los participantes, inmediatamente después de los talleres educativos, demostrando un aumento en el conocimiento sobre la enfermedad. Tres meses después de la intervención educativa, no hubo cambio estadísticamente significativo, demostrando que la retención del aprendizaje fue efectiva hasta el punto de perdurar en ese intervalo de tiempo. Conclusiones. En este estudio, el intervalo de tiempo entre la aplicación del cuestionario y la realización del recurso educativo no promovió la pérdida de aprendizaje de los participantes.

Palabras clave. Diabetes Mellitus; Educación para la salud; cuidados personales

Trabalho realizado na Universidade Iguaçu - UNIG, Nova Iguaçu-RJ, Brasil.

\section{INTRODUÇÃO}

O Diabetes Mellitus (DM) é classificado como uma Doença Crônica Não Transmissível (DCNT) de origem multifatorial e não infecciosa que causa grandes prejuízos à qualidade de vida dos idosos, sendo considerado um sério problema de saúde pública, sobretudo nos países em desenvolvimento ${ }^{1,2}$.

O envelhecimento da população brasileira, potencializado pela implementação de políticas públicas de saúde e pelo declínio da mortalidade infantil, e dissociado de melhorias no padrão de vida da população, contribuíram para o aumento da prevalência das doenças crônicas não transmissíveis, dentre elas o $\mathrm{DM}^{3}$. 
Conforme dados do Instituto Brasileiro de Geografia e Estatística - IBGE (2010), o quantitativo de idosos brasileiros poderá ultrapassar os 30 milhões de pessoas nos próximos 20 anos, o que corresponde aproximadamente $13 \%$ da população geral ${ }^{4}$.

Devido à importância dada pela comunidade científica à avaliação da qualidade de vida e levando-se em conta as mudanças dos perfis demográficos, socioeconômicos e de morbidade da população brasileira, é imprescindível a realização de estudos voltados à população idosa, visando reduzindo o deterioramento oriundo do DM e reduzir a demanda aos serviços de saúde ${ }^{5}$.

Neste contexto, acredita-se que a educação em saúde seja a principal ferramenta capaz de empoderar e promover mudanças de atitudes nos pacientes acometidos pela doença em questão, minimizando os desfechos negativos da mesma. Objetiva-se fornecer ao indivíduo meios de mudança de práticas e comportamentos individuais, desenvolvendo nele uma independência responsável na prevenção de doenças ${ }^{6,7}$.

Entretanto, para sua efetiva implantação, torna-se necessário o envolvimento de três agentes preferenciais: os profissionais de saúde prezando pela prevenção e promoção da saúde tal como prezam pelos cuidados curativos; os gestores promovendo suporte aos primeiros; e a população demandante da aquisição de conhecimentos, para que possa obter maior independência nos cuidados individuais e coletivos $^{8}$. 
Desta forma, tendo em vista que o envelhecimento é um processo de enfraquecimento das funcionalidades orgânicas que pode vir acompanhado de muitas doenças crônicas, dentre elas o Diabetes Mellitus (DM), torna-se necessária a adoção de estratégias, por parte dos gestores e profissionais de saúde, capazes de prevenir, controlar e/ou qualificar o cuidado prestado aos pacientes acometidos por esta doença.

O objetivo dessa pesquisa foi analisar os efeitos da intervenção educativa em idosos portadores de DM, atendidos no Hospital da Posse do município de Nova Iguaçu, visando ampliar o conhecimento dos mesmos sobre o cuidado integral e interdisciplinar para o controle ou prevenção da doença.

\section{MÉTODO}

\section{Amostra}

Trata-se de um projeto de intervenção com aplicação de questionário, distribuição de folders explicativos e oficinas de educação em saúde junto aos pacientes com diagnóstico de DM atendidos no Hospital Geral de Nova Iguaçu, no período de outubro de 2019 a março de 2020.

O projeto recebeu aprovação do Comitê de Ética da Universidade Iguaçu, conforme CAAE 13529219.8.0000.8044 e todos os pacientes foram orientados quanto aos riscos e benefícios da pesquisa. 


\section{Procedimento}

A intervenção aconteceu da seguinte forma:

$1^{\circ}$ momento: aplicação de questionário visando avaliar o conhecimento prévio dos pacientes acerca características da doença, fatores de risco, formas de controle e tratamento adequado da doença

$2^{\circ}$ momento: realização de oficina de educação em saúde, com palestra sobre a doença, na sala de espera.

$3^{\circ}$ momento: aplicação do mesmo questionário, logo após a palestra, visando avaliar a retenção do aprendizado a curto prazo.

$4^{\circ}$ momento: após 3 meses, contatar via telefone os mesmos pacientes e aplicar novamente 0 mesmo questionário para identificação da retenção do conhecimento a médio prazo.

\section{Análise Estatística}

O Teste de McNemar foi empregado para analisar os achados de cada grupo, identificando a retenção do conhecimento pelos mesmos. Este teste trabalha com amostras pareadas para aumentar a precisão de uma comparação, podendo ser utilizados dados nominais e ordinais às amostras pareadas. Não são amostras independentes, mas sim correlacionadas com as repostas de cada integrante antes e após as intervenções em saúde, ou seja, pretende-se analisar a eficiência relacionada ao aprendizado antes e após a intervenção, utilizando cada participante como seu próprio controle. 
O valor do X2 calculado pelo Teste de McNemar com correção de Yates avaliou a alteração nas respostas antes e após as intervenções. A correção visou obter resultados mais conservadores, reduzindo o valor de $X$ e demonstrando que a hipótese nula não será rejeitada com tanta frequência como o X sem correção.

Os valores encontrados acima do $\mathrm{X} 2$ crítico, que é igual a 3,841 , permitem a rejeição da hipótese nula demonstrando que houve mudança significante de respostas após as intervenções.

\section{RESULTADOS}

A Tabela 1 apresenta as características demográficas dos portadores de diabetes participantes das intervenções educativas em saúde.

Desta forma, conclui-se que a maioria da população pertencia ao sexo feminino $(n=93 ; 60,8 \%)$, com faixa etária entre 71 e 75 anos $(n=55 ; 36,0 \%)$, solteiros $(n=81 ; 52,9 \%)$, raça branca $(n=71 ; 46,4 \%)$, alfabetizados $(n=114 ; 74,5 \%)$, não etilistas $(n=139 ; 90,8 \%)$, não tabagistas $(n=137$; $89,5 \%)$, sedentários $(n=116 ; 75,8 \%)$, não consomem açúcar $(n=75 ; 49,0 \%)$, tratam o diabetes por meio de medicações orais $(n=93 ; 60,8 \%)$ e fazem o uso regular das medicações no horário correto $(n=132 ; 86,3 \%)$.

Na Tabela 2 são apresentadas as respostas antes e após as palestras com o valor do $\mathrm{X}^{2}$ calculado pelo Teste de McNemar com correção de Yates. 
Tabela 1. Características demográficas dos portadores de diabetes participantes das intervenções educativas em saúde (n-153), Nova Iguaçu-RJ, 2019-2020.

\begin{tabular}{|c|c|c|}
\hline Variáveis & $\mathbf{n}$ & $\%$ \\
\hline \multicolumn{3}{|l|}{ Sexo } \\
\hline Feminino & 93 & 60,8 \\
\hline Masculino & 60 & 39,2 \\
\hline \multicolumn{3}{|l|}{ Faixa Etária } \\
\hline $65-70$ & 38 & 24,8 \\
\hline $71-75$ & 55 & 36,0 \\
\hline $76-80$ & 49 & 32,0 \\
\hline Maiores de 80 anos & 11 & 7,2 \\
\hline \multicolumn{3}{|l|}{ Estado Civil } \\
\hline Solteiro & 81 & 52,9 \\
\hline Casado & 52 & 34,0 \\
\hline Outros & 20 & 13,1 \\
\hline \multicolumn{3}{|l|}{ Raça } \\
\hline Negro & 69 & 45,1 \\
\hline Branco & 71 & 46,4 \\
\hline Pardo & 12 & 7,8 \\
\hline Indígena & 1 & 0,7 \\
\hline \multicolumn{3}{|l|}{ Escolaridade } \\
\hline Não Alfabetizado & 16 & 10,5 \\
\hline Alfabetizado & 114 & 74,5 \\
\hline Ensino Fundamental & 11 & 7,0 \\
\hline Ensino Médio & 9 & 6,0 \\
\hline Ensino Superior & 3 & 2,0 \\
\hline Etilismo (sim) & 14 & 9,2 \\
\hline Tabagismo (sim) & 16 & 10,5 \\
\hline Prática regular de atividades físicas (sim) & 37 & 24,2 \\
\hline \multicolumn{3}{|l|}{ Consumo de açúcar } \\
\hline Frequentemente & 38 & 24,8 \\
\hline Às vezes & 40 & 26,2 \\
\hline Nunca & 75 & 49,0 \\
\hline \multicolumn{3}{|l|}{ Tratamento do diabetes } \\
\hline Medicações orais & 93 & 60,8 \\
\hline Insulina & 51 & 33,3 \\
\hline Medicações orais e Insulina & 9 & 5,9 \\
\hline \multicolumn{3}{|l|}{ Uso regular das medicações } \\
\hline Usa na hora certa & 132 & 86,3 \\
\hline Às vezes esquece & 18 & 11,8 \\
\hline Não usa regularmente & 3 & 1,9 \\
\hline
\end{tabular}


Tabela 2. Comparativo das respostas dos entrevistados antes e após a intervenção educativa em saúde, Nova Iguaçu - RJ, 2019-2020.

\begin{tabular}{|c|c|c|c|c|}
\hline \multirow{5}{*}{$\begin{array}{c}\text { Pergunta } \\
\text { Você sabe o que é Diabetes Mellitus? }\end{array}$} & \multicolumn{3}{|c|}{ Respostas } & \multirow{5}{*}{$\begin{array}{c}\mathbf{X}^{2} \text { calculado } \\
4,17\end{array}$} \\
\hline & \multirow{3}{*}{$\begin{array}{l}\text { Antes da } \\
\text { palestra } \\
\text { Não }\end{array}$} & \multicolumn{2}{|c|}{$\begin{array}{l}\text { Depois } \quad \mathrm{da} \\
\text { palestra }\end{array}$} & \\
\hline & & Sim & Não & \\
\hline & & 6 & 0 & \\
\hline & Sim & 147 & 0 & \\
\hline \multirow{4}{*}{$\begin{array}{l}\text { Você sabe quais são os sinais e sintomas do Diabetes } \\
\text { Mellitus? }\end{array}$} & & \multicolumn{2}{|c|}{$\begin{array}{l}\text { Depois da } \\
\text { palestra }\end{array}$} & \\
\hline & $\begin{array}{l}\text { Antes da } \\
\text { palestra }\end{array}$ & Sim & Não & \multirow{3}{*}{24,04} \\
\hline & Não & 26 & 16 & \\
\hline & Sim & 111 & 0 & \\
\hline \multirow{4}{*}{$\begin{array}{l}\text { O consumo excessivo de açúcar é o principal fator de } \\
\text { risco para Diabetes Mellitus? }\end{array}$} & & \multicolumn{2}{|c|}{$\begin{array}{l}\text { Depois da } \\
\text { palestra }\end{array}$} & \\
\hline & $\begin{array}{l}\text { Antes da } \\
\text { palestra }\end{array}$ & Sim & Não & \multirow{3}{*}{70,01} \\
\hline & Não & 72 & 21 & \\
\hline & Sim & 60 & 0 & \\
\hline \multirow{4}{*}{$\begin{array}{l}\text { O antecedente familiar é o principal fator de risco para } \\
\text { Diabetes Mellitus? }\end{array}$} & & \multicolumn{2}{|c|}{$\begin{array}{l}\text { Depois da } \\
\text { palestra }\end{array}$} & \\
\hline & $\begin{array}{l}\text { Antes da } \\
\text { palestra }\end{array}$ & Sim & Não & \multirow{3}{*}{70,01} \\
\hline & Não & 0 & 60 & \\
\hline & Sim & 21 & 72 & \\
\hline \multirow{4}{*}{$\begin{array}{l}\text { A melhor forma de prevenir o Diabetes Mellitus é com } \\
\text { alimentação equilibrada e prática de exercícios } \\
\text { regulares }\end{array}$} & & $\begin{array}{l}\text { Depc } \\
\text { pales }\end{array}$ & da & \multirow{4}{*}{38,03} \\
\hline & $\begin{array}{l}\text { Antes da } \\
\text { palestra }\end{array}$ & Sim & Não & \\
\hline & Não & 40 & 0 & \\
\hline & Sim & 113 & 0 & \\
\hline \multirow{4}{*}{$\begin{array}{l}\text { A melhor forma de prevenir o Diabetes Mellitus é } \\
\text { apenas não comer doces }\end{array}$} & & $\begin{array}{l}\text { Depc } \\
\text { pales }\end{array}$ & $\mathrm{da}$ & \\
\hline & $\begin{array}{l}\text { Antes da } \\
\text { palestra }\end{array}$ & Sim & Não & \multirow{3}{*}{26,04} \\
\hline & Não & 0 & 125 & \\
\hline & Sim & 0 & 28 & \\
\hline \multirow{4}{*}{$\begin{array}{l}\text { A melhor forma de prevenir o Diabetes Mellitus é } \\
\text { apenas fazendo exames de rotina }\end{array}$} & & $\begin{array}{l}\text { Depc } \\
\text { pales }\end{array}$ & $\mathrm{da}$ & \\
\hline & $\begin{array}{l}\text { Antes da } \\
\text { palestra }\end{array}$ & Sim & Não & \multirow{3}{*}{10,08} \\
\hline & Não & 0 & 141 & \\
\hline & Sim & 0 & 12 & \\
\hline \multirow{4}{*}{$\begin{array}{l}\text { A forma mais segura de controlar meu diabetes é } \\
\text { tomando a medicação corretamente }\end{array}$} & & $\begin{array}{l}\text { Depc } \\
\text { pales }\end{array}$ & $\mathrm{da}$ & \\
\hline & $\begin{array}{l}\text { Antes da } \\
\text { palestra }\end{array}$ & Sim & Não & \multirow{3}{*}{5,14} \\
\hline & Não & 7 & 1 & \\
\hline & Sim & 145 & 0 & \\
\hline \multirow{4}{*}{$\begin{array}{l}\text { A forma mais segura de controlar meu diabetes é } \\
\text { praticando exercícios físicos }\end{array}$} & & $\begin{array}{l}\text { Depc } \\
\text { pales }\end{array}$ & $\mathrm{da}$ & \\
\hline & $\begin{array}{l}\text { Antes da } \\
\text { palestra }\end{array}$ & Sim & Não & \multirow{3}{*}{4,17} \\
\hline & Não & 0 & 146 & \\
\hline & Sim & 1 & 6 & \\
\hline
\end{tabular}


Houve mudança significante nas respostas dos participantes das oficinas educativas, pois todos os valores de $X^{2}$ calculados estão acima do $X^{2}$ crítico, que é igual a 3,841 . Analisando as respostas, mais pessoas ficaram sabendo o que é o diabetes mellitus, quais são os sinais e sintomas, fatores de risco e as formas mais eficazes de prevenção e controle. Pode-se deduzir que as oficinas educativas cumpriram o seu objetivo de educar os participantes.

Três meses após as oficinas educativas, os participantes foram contatados via telefone para responder às mesmas perguntas do questionário para comparação com as respostas dadas após a oficina educativa. A Tabela 3 correlaciona as respostas logo após a palestra e três meses depois com o valor do $\mathrm{X}^{2}$ calculado pelo Teste de McNemar com correção de Yates.

As mudanças das respostas dos pacientes três meses após a intervenção educativa em saúde não tiveram significância estatística, pois todos os valores do $\mathrm{X}^{2}$ calculados estão abaixo do $X^{2}$ crítico $(3,841)$. Isso significa que a retenção do aprendizado foi eficaz a ponto de perdurar até três meses após a intervenção educativa em saúde. 
Tabela 3. Comparativo das respostas dos entrevistados antes e após a intervenção educativa em saúde, Nova Iguaçu - RJ, 2019-2020.

\begin{tabular}{|c|c|c|c|c|}
\hline Pergunta & \multicolumn{3}{|c|}{ Respostas } & $\begin{array}{l}\mathbf{X}^{2} \\
\text { calcul } \\
\text { ado }\end{array}$ \\
\hline \multirow{4}{*}{ Você sabe o que é Diabetes Mellitus? } & & depc & meses & \multirow{4}{*}{1} \\
\hline & $\begin{array}{l}\text { Logo após a } \\
\text { palestra }\end{array}$ & Sim & Não & \\
\hline & Não & 1 & 0 & \\
\hline & Sim & 152 & 0 & \\
\hline \multirow{4}{*}{$\begin{array}{l}\text { Você sabe quais são os sinais e sintomas do Diabetes } \\
\text { Mellitus? }\end{array}$} & & 3 & meses & \\
\hline & $\begin{array}{l}\text { Logo após a } \\
\text { palestra }\end{array}$ & Sim & Não & \multirow{3}{*}{3,2} \\
\hline & Não & 5 & 16 & \\
\hline & Sim & 132 & 0 & \\
\hline \multirow{4}{*}{$\begin{array}{l}\text { O consumo excessivo de açúcar é o principal fator de } \\
\text { risco para Diabetes Mellitus? }\end{array}$} & & $\begin{array}{l}3 \\
\text { depc }\end{array}$ & meses & \\
\hline & $\begin{array}{l}\text { Logo após a } \\
\text { palestra }\end{array}$ & Sim & Não & \multirow{3}{*}{2,25} \\
\hline & Não & 0 & 21 & \\
\hline & Sim & 128 & 4 & \\
\hline \multirow{4}{*}{$\begin{array}{l}\text { O antecedente familiar é o principal fator de risco para } \\
\text { Diabetes Mellitus? }\end{array}$} & & $\begin{array}{l}3 \\
\text { depc }\end{array}$ & meses & \\
\hline & $\begin{array}{l}\text { Logo após a } \\
\text { palestra }\end{array}$ & Sim & Não & \multirow{3}{*}{2,25} \\
\hline & Não & 4 & 128 & \\
\hline & Sim & 21 & 0 & \\
\hline \multirow{4}{*}{$\begin{array}{l}\text { A melhor forma de prevenir o Diabetes Mellitus é com } \\
\text { alimentação equilibrada e prática de exercícios } \\
\text { regulares }\end{array}$} & & 3 & meses & \\
\hline & $\begin{array}{l}\text { Logo após a } \\
\text { palestra }\end{array}$ & Sim & Não & \multirow{3}{*}{1,33} \\
\hline & Não & 0 & 0 & \\
\hline & Sim & 150 & 3 & \\
\hline \multirow{4}{*}{$\begin{array}{l}\text { A melhor forma de prevenir o Diabetes Mellitus é } \\
\text { apenas não comer doces }\end{array}$} & & 3 & meses & \\
\hline & $\begin{array}{l}\text { Logo após a } \\
\text { palestra }\end{array}$ & Sim & Não & \multirow{3}{*}{1,33} \\
\hline & Não & 3 & 150 & \\
\hline & Sim & 0 & 0 & \\
\hline \multirow{4}{*}{$\begin{array}{l}\text { A melhor forma de prevenir o Diabetes Mellitus é } \\
\text { apenas fazendo exames de rotina }\end{array}$} & & depc & meses & \\
\hline & $\begin{array}{l}\text { Logo após a } \\
\text { palestra }\end{array}$ & Sim & Não & \multirow{3}{*}{0,00} \\
\hline & Não & 0 & 153 & \\
\hline & Sim & 0 & 0 & \\
\hline \multirow{4}{*}{$\begin{array}{l}\text { A forma mais segura de controlar meu diabetes é } \\
\text { tomando a medicação corretamente }\end{array}$} & & 3 & meses & \\
\hline & $\begin{array}{l}\text { Logo após a } \\
\text { palestra }\end{array}$ & Sim & Não & \multirow{3}{*}{1,33} \\
\hline & Não & 0 & 1 & \\
\hline & Sim & 149 & 3 & \\
\hline \multirow{4}{*}{$\begin{array}{l}\text { A forma mais segura de controlar meu diabetes é } \\
\text { praticando exercícios físicos }\end{array}$} & & $\begin{array}{l}3 \\
\text { depc }\end{array}$ & meses & \\
\hline & $\begin{array}{l}\text { Logo após a } \\
\text { palestra }\end{array}$ & Sim & Não & \multirow{3}{*}{1,33} \\
\hline & Não & 3 & 149 & \\
\hline & Sim & 1 & 0 & \\
\hline
\end{tabular}




\section{DISCUSSÃO}

Os pacientes entrevistados apresentaram pouco conhecimento sobre a DM, comprometendo o autocuidado e - controle da doença, corroborando com os achados encontrados em outros estudos sobre o tema ${ }^{8-10}$. Tendo em vista que o conhecimento é uma das variáveis que podem modificar as atitudes de aceitação do indivíduo frente ao manejo do Diabetes Mellitus, torna-se fundamental investir esforços para ampliar essa estratégia no âmbito dos serviços de saúde ${ }^{9,10}$.

Neste sentido, diferentes estudos sobre educação em saúde para pacientes com DM, vêm demonstrando a importância da inserção e envolvimentos das diferentes categorias profissionais de saúde para a qualificação do tratamento, bem como de diferentes ferramentas lúdicas e pedagógicas para apoiar o processo educativo ${ }^{11-13}$.

Desta forma, outras estratégias educativas podem ser testadas, em função do perfil dos pacientes e dos profissionais envolvidos, bem como dos recursos e período de tempo disponíveis para a realização das mesmas. Experiências como educação interativa, intervenção educativa comunitária, jogos em grupos operativos, seminários educativos, visitas domiciliares, conferências educativas, atividades práticas e realização de exames, relato de casos, teoria das representações sociais e colônia educativa, são algumas experiências descritas em literatura, que se mostraram efetivas para gerar modificações significantes no estilo de vida dos pacientes envolvidos ${ }^{14}$. 
Por outro lado, vêm sendo apontadas limitações no que tange a capacitação dos profissionais para realizar as ações de educação em saúde, demonstrando que tal competência deve ser reconhecida e formalizada como conteúdo do trabalho de educação permanente dos serviços das diferentes categorias profissionais ${ }^{15}$. Devendo então constituir-se como um processo dinâmico e contínuo, que abranja o maior número de pacientes possível e não apenas uma pequena parcela da população.

Estudo realizado em um serviço de atenção primária de uma cidade do interior paulista comprovou mais uma vez o potencial da educação em saúde para o tratamento em DM, uma vez que a permanência do conhecimento perdurou até 5 anos do término de um programa educativo em saúde ${ }^{16}$. Nosso estudo demonstrou a apreensão do conhecimento após 3 meses da estratégia de educação em saúde e muitos pacientes se sentiram motivados para a mudança do comportamento.

Independente dos ganhos apresentados, deve-se atentar que a apreensão do conhecimento por si só, não será capaz de reverter a situação encontrada integralmente, sendo apenas uma peça, embora muito relevante, do quebra cabeça ora exposto.

O apoio da família e da rede de amigos apresenta-se como um eixo primordial para a conscientização das mudanças necessárias ao sucesso do tratamento, 
compartilhando hábitos de vida saldáveis e evitando o isolamento social ${ }^{17,18}$.

Ainda, torna-se necessária a intervenção sobre o ambiente, no sentido de ampliação das políticas públicas capazes de promover a adoção de hábitos saudáveis, além da reorientação dos serviços de saúde para além dos tratamentos clínicos e curativos ${ }^{14}$.

\section{CONCLUSÃO}

Conclui-se que a educação em saúde é relevante para prevenção, diagnóstico e melhor controle do diabetes mellitus, sendo o trabalho conjunto entre profissionais de saúde e população capaz de garantir eficácia das medidas educativas. Entretanto, ainda são necessários novos estudos visando identificar se, a longo prazo, o conhecimento adquirido conseguiu provocar alteração nos hábitos e práticas pessoais, repercutindo de fato na saúde do paciente.

\section{REFERÊNCIAS}

1. Haddad S. Desafios na produção do cuidado à saúde: da hipertensão arterial e do diabetes à doença renal crônica (Dissertação). São Paulo: Coordenadoria de Recursos Humanos da Secretaria de Estado da Saúde de São Paulo, 2016.

https://docs.bvsalud.org/biblioref/2019/09/1006170/samirahaddaddissertacaocompleta.pdf

2.IBGE. Síntese de indicadores sociais: uma análise das condições de vida da população brasileira. Ministério do Planejamento, Orçamento e gestão. Rio de Janeiro: IBGE, 2010. https://www.gov.br/mdh/pt$\mathrm{br} /$ navegue-por-temas/politicas-para-mulheres/arquivo/areaimprensa/documentos-1/SintIndicadoresSociais 2010 Embargo.pdf 3.International Diabetes Federation. Complicações do diabetes e educação. Diabetes Clin 2002;6:217-20.

4.International Diabetes Federation (IDF). Diabetes Atlas. 7ed. Brussels: IDF; 2015. 
5.Borges JES, Camelier AA, Oliveira LVF, Brandão GS. Qualidade de vida de idosos hipertensos e diabéticos da comunidade: um estudo observacional. Rev Pesqui Fisioter 2019;9:74-84. https://doi.org/10.17267/2238-2704rpf.v9i1.2249

6. Falkenberg MB, Mendes TPL, Moraes EP, Souza EM. Educação em saúde e educação na saúde: conceitos e implicações para a saúde coletiva. Ciênc Saúde Colet 2014;19:847-52. https://doi.org/10.1590/1413-81232014193.01572013

7.Barreto TCPP, Barreto APP, Reis J, Santos RA. Diabetes Mellitus: knowledge and attitudes, collaborating for individual and social development of a reef community. Diabetol Metab Syndr 2015; 7(suppl 1):A174. https://doi.org/10.1186/1758-5996-7-S1-A174

8.Janini JP, Bessler D, Vargas AB. Educação em saúde e promoção da saúde: impacto na qualidade de vida do idoso. Saúde em Debate 2015;39:480-90.

https://doi.org/10.1590/0103-

\section{5}

9. Oliveira KCS, Zanetti ML. Knowledge and attitudes of patients with diabetes mellitus in a primary health care system. Rev Esc Enferm USP 2011;45:862-8. https://doi.org/10.1590/S0080-62342011000400010 10.Rodriguez FFL, Santos MA, Teixeira CRS, Gonela JT, Zanetti ML. Relationship between knowledge, attitude, education and duration of disease in individuals with diabetes mellitus. Acta Paul Enferm 2012;25:284-90.

https://doi.org/10.1590/S0103-

21002012000200020

11. Oliveira KCS, Zanetti ML. Knowledge and attitudes of patients with diabetes mellitus in a primary health care system. Rev Esc Enferm USP 2011;45:862-8. https://doi.org/10.1590/S0080-62342011000400010 12.Santos FS, Oliveira KR, Colrt CF. Adesão ao tratamento medicamentoso pelos portadores de diabetes mellitus, atendidos em uma Unidade básica de saúde no município de Ijuí: um estudo exploratório. Rev Cienc Farm Básica Apl 2010;31:223-7. https://rcfba.fcfar.unesp.br/index.php/ojs/article/view/369/367

13. Coelho MCVS, Almeida CAPL, Da Silva ARV, Moura LKB, Feitosa LGGC, Nunes B. Formação em educação em diabetes: significados atribuídos por enfermeiros da atenção primária. Rev Bras Enferm 2018;71:1707-14. https://doi.org/10.1590/0034-7167-2017-0792 14.Silva ARVD, Macedo SF, Vieira NFC, Pinheiro PNC, Damasceno MMC. Educação em saúde a portadores de diabetes mellitus tipo 2: revisão bibliográfica. Rev Rene 2009;10:146-51. https://www. redalyc.org/articulo.oa?id=324027967018

15.Salci MA, Meirelles BHS, Silva DMGVD. Educação em saúde para prevenção das complicações crônicas do diabetes mellitus na atenção primária. Escola Anna Nery 2018;22:e20170262. https://doi.org/10.1590/2177-9465-EAN-2017-0262

16.Chagas IA, Camilo J, Santos MA, Rodrigues FFL, Arrelias CCA, Teixeira CRS, et al. Conhecimento de pacientes com diabetes sobre tratamento após cinco anos do término de um programa educativo. Rev Escola Enferm USP 2013;47:1141-6. https://doi.org/10.1590/S0080-623420130000500018 
17. Costa JDA, Balga RSM, Alfenas RDCG, Cotta RMM. Promoção da saúde e diabetes: discutindo a adesão e a motivação de indivíduos diabéticos participantes de programas de saúde. Cienc Saúde Colet 2011;16:2001-9.

http://www.cienciaesaudecoletiva.com.br/artigos/promocao-da-

saude-e-diabetes-discutindo-a-adesao-e-a-motivacao-de-individuosdiabeticos-participantes-de-programas-de-saude/2467

18.Santos MAD, Péres DS, Zanetti ML, Otero LM, Teixeira CRDS. Programa de educação em saúde: expectativas e benefícios percebidos por pacientes diabéticos. Rev Enferm UERJ 2009;17:57-63. http://files.bvs.br/upload/S/0104-3552/2009/v17n1/a010.pdf 